\title{
Influence des zones agroécologiques sur les paramètres biologiques de Callosobruchus maculatus (Coleoptera-Bruchidea), ravageurs des graines du niébé (Vigna unguiculata Walp.) au Niger
}

\author{
D. A. MOUMOUNI ${ }^{1 *}$, A. DOUMMA ${ }^{1}$ et M. SEMBENE ${ }^{2}$ \\ ${ }^{1}$ Université Abdou Moumouni de Niamey-Faculté des Sciences et Techniques ; BP. 10662 Niamey, Niger. \\ ${ }^{2}$ Université Cheick Anta Diop de Dakar, Faculté des Sciences et Techniques, \\ Laboratoire de Biologie et de Gestion des Populations (FST), Dakar, Sénégal. \\ *Auteur correspondant ; E-mail: admoumouni@yahoo.fr
}

\section{RESUME}

$\mathrm{Au}$ cours de cette étude, les paramètres biologiques de Callosobruchus maculatus provenant de différentes zones agro-écologiques du Niger sont déterminés. Pour cela, des souches de $C$. maculatus ont été collectées dans 4 localités représentant chacune une zone agro-écologique. L'analyse des résultats obtenus montre que quelle que soit la souche, la longévité des adultes de cette espèce ne dépasse guère 7 jours. Par contre, la fécondité est significativement plus importante pour les souches provenant de la zone sahélienne que pour celles provenant des autres zones agroécologiques. Par ailleurs, L'évolution des pontes montre que quelle que soit la souche, plus de $96 \%$ des œufs sont pondus pendant les quatre premiers jours de l'infestation. En outre, les souches se caractérisent par un taux de fertilité des œufs relativement important et une durée d'éclosion des œufs peu variable. La durée de développement est relativement plus élevée chez les souches de la zone du fleuve ( 33 jours) que chez celles de la zone saharienne et sahélienne ( 30 jours). Le taux de survie larvaire et le taux d'émergence sont plus importants pour les souches de la zone sahélo-soudanienne (Gaya) et sahélienne (Tarna) que pour celles originaires des zones saharo-sahélienne et saharienne.

Mots clés: Niébé (Vigna unguiculata), Callosobruchus maculatus, Paramètres biologiques, zones agroécologiques, Niger.

\section{INTRODUCTION}

Le niébé, Vigna unguiculata (L.) Walp.) est une légumineuse alimentaire dont les graines constituent la source de protéines la moins onéreuse pour la plupart des populations africaines. En effet, la production du niébé qui a été de 1773000 tonnes en 2010 connait une baisse en 2011 avec une production moyenne en tonne de 1517140 tonnes au Niger (RECA, 2012).

Cependant, la production du niébé, déjà tributaire par les aléas climatiques, est limitée par les pertes occasionnées par des Coléoptères Bruchidae qui rendent très difficile sa conservation après la récolte. En effet, les stades larvaires de ces insectes ravageurs se développent à l'intérieur des graines et consomment les réserves contenues dans les cotylédons (Glitho, 1990).

Les plus importants de ces ravageurs sont Bruchidius atrolineatus Pic. et Callosobruchus maculatus Fab. dont les adultes apparaissent dans les cultures à la fin de la saison des pluies et s'y reproduisent lors de la période de fructification du niébé (Alzouma et al., 1985).

Pendant l'entreposage du niébé, le taux d'infestation initial par les bruches est généralement inférieur à 5\% (Ouedraogo, 1991 ; Sanon et al., 2005). Ce taux passe à $30 \%$ après 1 mois, puis 80 à $100 \%$ en l'espace de 5 à 6 mois de stockage si aucune mesure de 
contrôle n'est entreprise (Glitho, 1990 ; Seck et al., 1992 ; Ouedraogo et al., 1996; Sanon et al., 1998). Par l'intermédiaire de sa forme non voilière, C. maculatus arrive à se maintenir dans les stocks durant toute la période de stockage contrairement à Bruchidius atrolineatus qui disparaît des stocks après le mois de décembre (Doumma, 1998; Zannou, 2000).

Dans le souci de pallier les pertes dues aux coléoptères bruchidae et particulièrement C. maculatus, plusieurs méthodes de lutte ont été développées (utilisation des plantes insecticides ou répulsives, des huiles essentielles, de cendre etc.).

Cependant, il est clairement admis que la mise au point d'une stratégie de lutte contre les insectes nécessite la connaissance préalable de leur bio-écologie qui, selon Lale et Vidal (2003), est influencée par les facteurs climatiques qui influencent leur développement et leur reproduction. Ceci est d'autant plus important que le Niger, un pays vaste $\left(1267000 \mathrm{~km}^{2}\right)$ renferme quatre (4) zones agro-écologiques différentes.
Le présent travail est une contribution à la connaissance de la biologie de $C$. maculatus, espèce de bruche responsable de l'essentiel des dégâts occasionnés en conditions de stockage au Niger. Il vise à étudier l'influence de l'origine géographique de la souche du ravageur sur ses capacités de reproduction.

\section{MATERIEL ET METHODES}

\section{Origine des souches de $\boldsymbol{C}$. maculatus}

Les différentes souches de $C$. maculatus utilisées dans les expériences proviennent de graines de niébé collectées auprès des producteurs à Tarna (13²7'47,6' 'N et $007^{\circ} 06^{\prime} 31,0^{\prime}$ ' $\mathrm{E}$ ) (Région de Maradi), à Gaya $\left(11^{\circ} 53^{\prime} 3 \mathrm{~N}\right.$ et $\left.3^{\circ} 26^{\prime} 57 \mathrm{E}\right)$ (Région de Dosso), à Ayérou ( $14^{\circ} 43^{\prime} 00^{\prime \prime} \mathrm{N}$ et $0^{\circ} 55^{\prime} 00^{\prime \prime} \mathrm{E}$ ) (Région de Tillabery) et à Kao $\left(15^{\circ} 53^{\prime} 53^{\prime \prime} \mathrm{N}\right.$ et $\left.005^{\circ} 48^{\prime} 11^{\prime \prime} \mathrm{E}\right)$ (Département de Tchintabaraden-Région de Tahoua). Ces quatre localités appartiennent chacune à l'une des zones agro écologiques du pays (Figure 1).

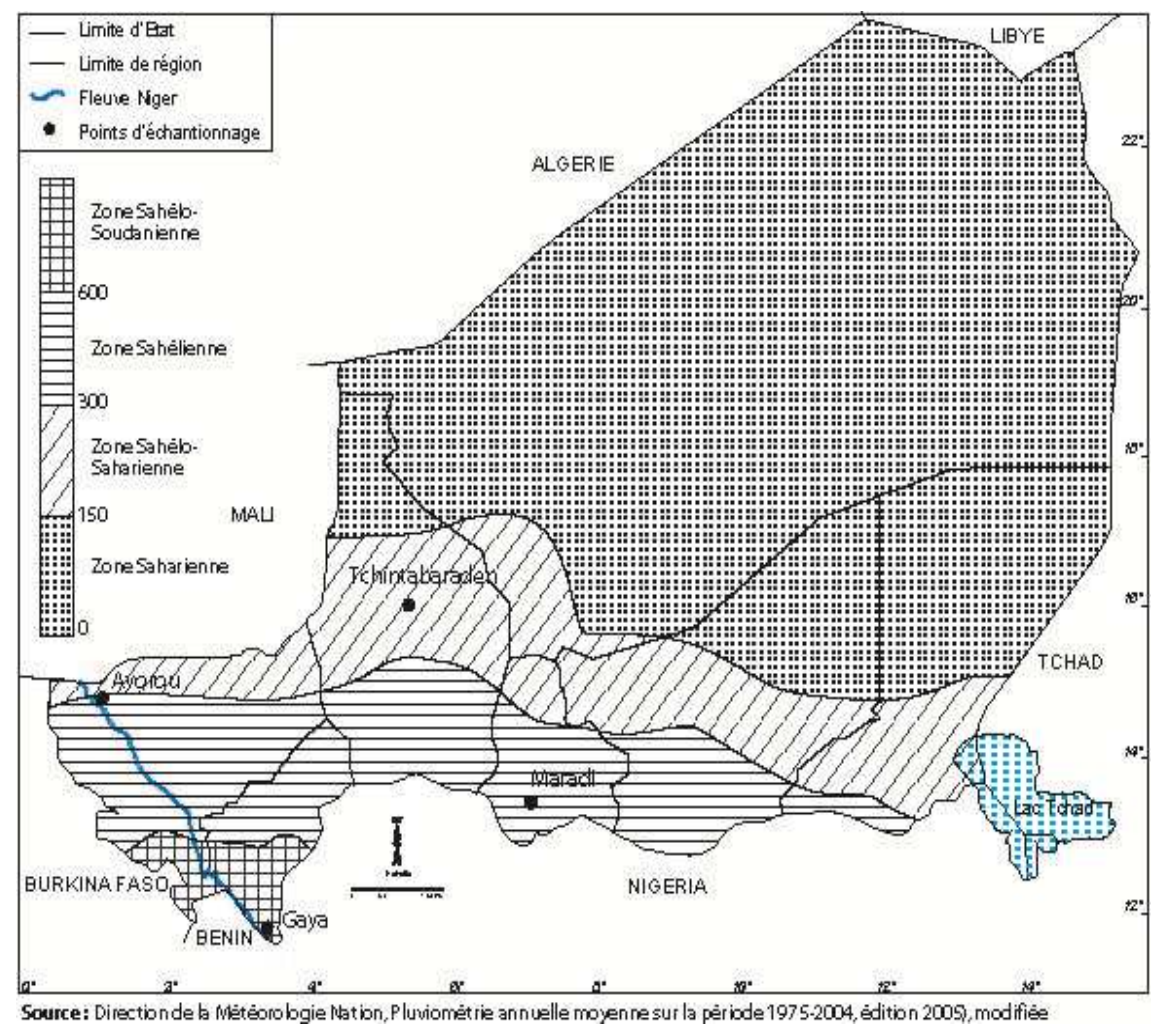

Figure 1: Carte du Niger montrant les zones climatiques du Niger et l'origine géographique des souches de C. maculatus. 
Dans chaque localité, quatre (04) échantillons de $3 \mathrm{~kg}$ de graines infestées de niébé sont prélevés des greniers des trois (3) producteurs. Ces échantillons ont ensuite été ramenés et conservés au laboratoire dans des boîtes parallélépipédiques en plexiglass (260×130×77 cm) jusqu'à l'émergence des adultes utilisés pour l'élevage.

\section{Élevage des insectes}

Selon l'origine géographique, les adultes de $C$. maculatus obtenus à partir des lots collectés dans les différentes localités ont été introduits dans un bocal contenant environ $100 \mathrm{~g}$ de graines saines de niébé de la variété TN 5-78. Quarante huit heures après, les insectes sont retirés et les graines contaminées laissées en incubation jusqu'à l'émergence des adultes qui ont plus tard été utilisés pour l'expérience.

\section{Méthodologie}

Pour étudier les paramètres biologiques (durée de vie, fécondité etc.) à l'exception de la durée d'éclosion, la méthodologie consiste à introduire un couple de C. maculatus dans une boîte de pétri contenant 10 graine saine. L'expérience est répétée vingt fois pour chacune des souches étudiées. $\mathrm{Au} 10^{\mathrm{e}}$ jour après la ponte (jours au cours duquel tous les œufs fertiles sont éclos), les œufs fertiles et les œufs stériles sont dénombrés. L'œuf stérile se distingue de l'œuf fertile par son aspect translucide sur une graine. Puis les graines sont conservées dans leurs boîtes jusqu'à l'émergence des adultes. A l'émergence, un suivi d'émergence des adultes est effectué et chaque jour les individus mâles et femelles émergés sont dénombrés dans chaque boîte. Le suivi d'émergence dure 10 jours. Pour déterminer la durée d'éclosion ou durée de développement embryonnaire, un suivi d'éclosion est réalisé. Premièrement des adultes de $C$. maculatus nouvellement émergés sont mis dans une boîte d'élevage contenant environ 150 à 200 graines saines. $24 \mathrm{~h}$ après les insectes sont retirées et les graines portant un seul œuf sont choisies. L'expérience consiste à repartir une graine portant un seul œuf dans une boîte de pétri. Au total 50 répartitions à été réalisés. Chaque jour les 50 graines sont observées et les œufs éclos sont notés. L'œuf éclos se distinct par une tâche noire à l'intérieur de celui-ci sur une graine. Le suivi d'éclosion dure 10 jours et les œufs non éclos à ce stade sont considérés stériles.

\section{Paramètres étudiés}

A l'issu des expériences précédente, les paramètres suivants sont déterminés:

- La durée de vie de l'adulte : C'est l'intervalle de temps entre l'émergence de l'insecte et sa mort;

- Le nombre d'œufs pondus par femelles (N) qui correspond au nombre total d'œufs pondus par femelle durant toute sa durée de vie.

- Le taux de fertilité : C'est le pourcentage d'œufs fertiles par rapport au nombre total d'œufs pondus;

- La durée de développement (T): c'est le temps qui sépare l'émission d'un œuf sur une graine et l'émergence de l'adulte;

- Le taux de survie larvaire (S) : C'est le pourcentage d'individus émergés par rapport au nombre total d'œufs fertiles;

- Le taux d'émergence (TE) : c'est le pourcentage d'individus émergés par rapport au nombre total d'œufs pondus;

- La durée du développement embryonnaire ou durée d'éclosion: c'est le temps entre l'émission d'un œuf sur une graine et le jour où celui-ci rentre dans la graine.

Le sex-ratio (R) correspond au rapport numérique entre les mâles et les femelles de la descendance.

\section{Traitements statistiques des résultats}

Toutes les données ont été soumises à l'analyse de variance et la séparation des moyennes a été faite soit selon le test de comparaison multiple de Duncan (données sur la fécondité et émergence des adultes), le test de Newman-Keuls ou le Test de Fisher ( $\mathrm{p}=$ 0.05) pour la comparaison deux à deux des souches.

\section{RESULTATS}

La durée de vie de $\boldsymbol{C}$. maculatus

Les résultats consignés dans le Tableau 1 montrent que la durée de vie de $C$. maculatus dépend du sexe et de l'origine géographique de la souche. En effet, la durée 
de vie moyenne a varié de $4,17 \mathrm{j}$ (Gaya) à $5,25 \mathrm{j}$ (Tarna) et est statistiquement différente (Tableau 1) d'une zone à une autre. Les souches de Tarna et d'Ayerou vivent beaucoup plus longtemps $(5,25 \pm 1,33$ jours pour la souche de Tarna et $4,67 \pm 0,88$ jours pour la souche d'Ayerou) que les souches de Gaya et Tchintabaraden $(4,17 \pm 1,07$ jours pour

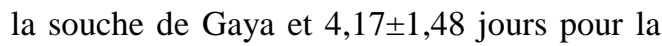
souche de Tchintabaraden).

Dans nos conditions expérimentales, la durée de vie des femelles est statistiquement (Tableau 1) la même quelque soit la zone agro-écologique considérée. Par contre, la durée de vie des mâles varie en fonction de l'origine géographique de la souche. Les mâles de C. maculatus provenant de Tarna sont caractérisés par une durée de vie relativement élevée alors que les mâles de la souche de Tchintabaraden se caractérisent par une durée de vie relativement courte (4, 17 $\pm 1,07$ jours).

\section{Evolution de la mortalité des adultes}

La Figure 2 indique l'évolution de la mortalité de $C$. maculatus dans les quatre localités. Il ressort que la mortalité des adultes commence au deuxième jour (J2) pour les souches de Tarna et de Tchintabaraden et débute à partir du troisième jour (J3) pour les souches de Gaya et d'Ayerou.

La mortalité est beaucoup plus étalée pour la souche de Tchintabaraden pour laquelle elle est allée jusqu'au $8^{\mathrm{e}}$ jour.

Toutefois, l'examen du graphique montre qu'à $6^{\mathrm{e}}$ jour, le taux de mortalité des adultes a varié de $90 \%$ (souche de Tchintabaraden) à 100\% (souche d'Ayérou).

\section{Etude de l'activité de ponte}

Variation du nombre d'œufs pondus par $C$. maculatus

Les résultats du Tableau 2 montrent que la fécondité des femelles de C. maculatus dépend de l'origine géographique des insectes. En effet, les résultats montrent une différence significative du nombre moyen d'œufs pondus en fonction de la zone agroécologique. Le nombre d'œufs pondus est plus important chez la souche provenant de Maradi (58,4 $\pm 20,76$ œufs par femelle) et plus faible chez la souche provenant de Gaya avec une moyenne d'œufs de 40,75 $\pm 14,99$ œufs par femelle. Par contre, le taux de fertilité des œufs ne semble pas varier en fonction de l'origine géographique de $C$. maculatus. En effet, quelle que soit la localité considérée, le taux de fertilité est supérieur à $94 \%$.

\section{Evolution de l'activité de pontes}

La Figure 3 montre que l'activité de ponte commence dès le premier jour dans les quatre souches. La souche de Tarna présente une activité de ponte relativement plus importante $(58,4 \pm 20,76$ œufs par femelle) par rapport aux souches provenant des autres localités (Figure 3).

Ces résultats montrent que quelle que soit la souche de $C$. maculatus considérée, les femelles déposent près de $96 \%$ de leurs œufs au cours des quatre premiers jours de vie (Tableau 3).

La Figure 3 montre que l'activité de ponte est liée à l'âge de la femelle. Ainsi, pour les souches de Tarna, Gaya et Ayerou, l'activité de ponte est maximale dès le premier jour (J1) de ponte et diminue progressivement pour devenir presque nulle à la fin de la vie de la femelle. La même tendance est observée pour la souche de Tchintabaraden, mais avec un pic observé au $2^{\mathrm{e}}$ jour de ponte (J2).

\section{Etude du développement de $C$. maculatus La durée de développement embryonnaire}

Le Tableau 4 montre que la durée du développement embryonnaire varie selon l'origine géographique de la souche de $C$. maculatus utilisée.

Elle est plus longue pour la souche de Tarna et Ayérou dont la durée moyenne de développement embryonnaire observée est voisine de $5 \mathrm{j}$. Par contre, elle est plus courte pour les souches de Gaya $(3,59 \pm 0,79 \mathrm{j})$ et de Tchintabaraden $(3,62 \pm 2,06 \mathrm{j})$.

\section{La durée de développement totale}

La durée totale de développement des adultes varie également selon les localités. Elle est beaucoup plus longue pour les souches de Gaya $(33,12 \pm 2,73 \mathrm{j})$ et Ayerou $(31,92 \pm 3,37 \mathrm{j})$ que pour les souches de Tarna $(30,63 \pm 3,60 \mathrm{j})$ et Tchintabaraden $(29,1 \pm 2,7 \mathrm{j})$ (Tableau 4).

En approchant la durée de développement embryonnaire et la durée 
totale de développement de l'insecte, il apparait qu'il n'y a pas de relation entre ces deux paramètres. En effet, en examinant le Tableau 4, on constate que la souche d'Ayérou a une durée totale de développement plus courte que la souche de Gaya chez laquelle la durée de développement embryonnaire est relativement courte.

Etude du développement postembryonnaire

La survie larvaire et l'émergence des adultes

Les résultats consignés dans le

Tableau 5 montrent que, quelle que soit la localité considérée, plus de $65 \%$ des larves ayant pénétré dans les graines ont donné des adultes. Toutefois, les taux de survie observés dépendent de la souche considérée. En effet, la survie larvaire observée au niveau des souches de Tarna $(90,94 \pm 0,82 \%)$ et de Gaya $(83,42 \pm 0,53 \%)$ est plus élevée que celle observée au niveau des souches d'Ayérou $(70,55 \pm 0,13 \%)$ et de Tchintabaraden
$(67,42 \pm 0,26 \%)$. Cette variation de la survie larvaire en fonction de l'origine se traduit par une variation des taux d'émergence des adultes (Tableau 5). En effet, le taux d'émergence enregistré pour les souches de Tchintabaraden $(66,16 \pm 0,43 \%)$ et Ayérou $(66,98 \pm 0,35 \%)$ est plus faible que celui enregistré pour les souches de Gaya $(79,63 \pm 0,13 \%)$ et Tarna $(89,38 \pm 0,65 \%)$. Par ailleurs quelle que soit l'origine de la souche, il ressort que plus de $80 \%$ des adultes émergent dans les 8 premiers jours de suivi (Tableau 6).

\section{Variation du sex-ratio}

Le sex-ratio qui est le rapport entre les descendants femelles et les descendants mâles (Tableau 7) a varié d'une souche à l'autre. Il est en faveur des femelles pour les souches de Gaya $(1,14)$ et d'Ayerou $(1,05)$. Par contre pour les souches de Maradi $(0,78)$ et de Tchintabaraden $(0,81)$, il est en faveur des mâles.

Tableau 1: Variation de la durée de vie moyenne de C. maculatus selon le sexe et l'origine agroecologique.

\begin{tabular}{lccc}
\hline $\begin{array}{l}\text { Origine des } \\
\text { souches }\end{array}$ & $\begin{array}{c}\text { Durée de vie moyenne } \\
\text { (jours) }\end{array}$ & $\begin{array}{c}\text { Durée de vie des } \\
\text { femelles }\end{array}$ & $\begin{array}{c}\text { Durée de vie des } \\
\text { mâles }\end{array}$ \\
\hline Maradi & $5,25 \pm 1,33 \mathrm{a}$ & $4,8 \pm 1,218 \mathrm{a}$ & $5,70 \pm 1,455 \mathrm{a}$ \\
Gaya & $4,17 \pm 1,072 \mathrm{~b}$ & $4,35 \pm 1,226 \mathrm{a}$ & $4,00 \pm 0,918 \mathrm{~b}$ \\
Ayerou & $4,67 \pm 0,886 \mathrm{c}$ & $4,45 \pm 0,851 \mathrm{a}$ & $4,90 \pm 0,912 \mathrm{c}$ \\
Tchintabaraden & $4,17 \pm 1,489 \mathrm{~b}$ & $4,95 \pm 1,791 \mathrm{a}$ & $3,40 \pm 1,188 \mathrm{~b}$ \\
\hline \multicolumn{2}{c}{$\begin{array}{l}\text { Dans une même colonne, les moyennes suivies par la même lettre ne sont pas significativement différentes au seuil } \\
\text { de 5\% (Test de Newman-Keuls). }\end{array}$}
\end{tabular}

Tableau 2: Activité de ponte et taux de fertilité des œufs de C. maculatus en fonction de l'origine de la souche.

\begin{tabular}{lccc}
\hline Origine des souches & $\begin{array}{c}\text { Nombre moyen d'œufs } \\
\text { ( } \pm \text { Ecart type) }\end{array}$ & $\begin{array}{c}\text { Nombre moyen } \\
\text { d'œufs éclos /femelle } \\
( \pm \text { Ecart type })\end{array}$ & $\begin{array}{c}\text { Taux de fertilité } \\
(\%)\end{array}$ \\
\hline Maradi & $58,4 \pm 20,76 \mathrm{a}$ & $57,4 \pm 22,32 \mathrm{a}$ & 98,28 \\
Gaya & $40,75 \pm 14,99 \mathrm{~b}$ & $38,9 \pm 15,53 \mathrm{~b}$ & 95,46 \\
Ayerou & $42,4 \pm 17 \mathrm{~b}$ & $40,25 \pm 17,24 \mathrm{~b}$ & 94,92 \\
Tchintabaraden & $43 \pm 15,05 \mathrm{~b}$ & $42,2 \pm 16,26 \mathrm{~b}$ & 98,13
\end{tabular}

Dans une même colonne, les valeurs suivies par la même lettre alphabétique ne sont pas significativement différentes au seuil de 5\% (Test de Newman-Keuls). 
Tableau 3: Pourcentage cumulé d'œufs pondus au cours de la vie de la femelle.

\begin{tabular}{lccccc}
\hline $\begin{array}{l}\text { Origine des } \\
\text { souches }\end{array}$ & $\begin{array}{c}\text { \% } \\
\mathbf{1}^{\text {er }} \text { jour }\end{array}$ & $\begin{array}{c}\text { \% } \\
\mathbf{2} \text { premiers } \\
\text { jours }\end{array}$ & $\begin{array}{c}\mathbf{\%} \text { premiers } \\
\text { jours }\end{array}$ & $\begin{array}{c}\text { \% } \\
\text { 4 premiers } \\
\text { jours }\end{array}$ & $\begin{array}{c}\text { \% reste de la } \\
\text { vie de la } \\
\text { femelle }\end{array}$ \\
\hline Maradi & 32,10 & 58,30 & 83,98 & 96,74 & 3,26 \\
Gaya & 40,24 & 63,43 & 87,85 & 97,18 & 2,82 \\
Ayérou & 37,73 & 63,67 & 81,36 & 98,59 & 1,41 \\
Tchinta & 28,72 & 61,39 & 74,41 & 96,87 & 3,13 \\
\hline
\end{tabular}

Tableau 4: Variation de la durée de développement de C. maculatus en fonction des localités.

\begin{tabular}{lcc}
\hline Origine des souches & $\begin{array}{c}\text { Durée de développement } \\
\text { embryonnaire }\end{array}$ & $\begin{array}{c}\text { Durée de développement } \\
\text { totale }\end{array}$ \\
\hline Maradi & $4,97 \pm 1,29 \mathrm{a}$ & $30,63 \pm 3,60 \mathrm{a}$ \\
Gaya & $3,59 \pm 0,79 \mathrm{~b}$ & $33,12 \pm 2,73 \mathrm{~b}$ \\
Ayerou & $4,91 \pm 1,56 \mathrm{a}$ & $31,92 \pm 3,37 \mathrm{~b}$ \\
Tchintabaraden & $3,62 \pm 2,06 \mathrm{~b}$ & $29,1 \pm 2,7 \mathrm{a}$ \\
\hline
\end{tabular}

Dans une même colonne, les moyennes suivies par la même lettre ne sont pas significativement différentes au seuil de 5\% (Test de Newman-Keuls).

Tableau 5: Taux de survie larvaire et taux d'émergence de $C$. maculatus en fonction de l'origine de la souche.

\begin{tabular}{lcccc}
\hline Localités & $\begin{array}{c}\text { Nombre moyen } \\
\text { d'oufs fertiles pondus } \\
( \pm \text { Ecart type })\end{array}$ & $\begin{array}{c}\text { Nombre moyen } \\
\text { d'adultes émergés } \\
( \pm \text { Ecart type })\end{array}$ & $\begin{array}{c}\text { Taux de survie } \\
\text { larvaire }(\mathbf{S}) \\
(\%)\end{array}$ & $\begin{array}{c}\text { Taux } \\
\text { d'émergence } \\
(\mathbf{T E})\end{array}$ \\
\hline Maradi & $57,4 \pm 22,32 \mathrm{a}$ & $52,2 \pm 16,28 \mathrm{~b}$ & $90,94 \pm 0,82 \mathrm{a}$ & $89,38 \pm 0,65 \mathrm{a}$ \\
Gaya & $38,9 \pm 15,53 \mathrm{~b}$ & $32,45 \pm 10,23 \mathrm{a}$ & $83,42 \pm 0,53 \mathrm{a}$ & $79,63 \pm 0,13 \mathrm{a}$ \\
Ayerou & $40,25 \pm 17,24 \mathrm{~b}$ & $28,4 \pm 11,50 \mathrm{a}$ & $70,55 \pm 0,13 \mathrm{~b}$ & $66,98 \pm 0,35 \mathrm{~b}$ \\
Tchinta & $42,2 \pm 16,26 \mathrm{~b}$ & $28,45 \pm 15,71 \mathrm{a}$ & $67,42 \pm 0,26 \mathrm{~b}$ & $66,16 \pm 0,43 \mathrm{~b}$ \\
\hline \multicolumn{4}{c}{ Dans une même colonne, les moyennes suivies par la même lettre ne sont pas significativement différentes au seuil de } \\
5\% (Test de Newman-Keuls).
\end{tabular}

Tableau 6 : Pourcentage d'adultes émergeants de $C$. maculatus au cours de la période de suivi.

\begin{tabular}{lcccc}
\hline Origine des souches & $\mathbf{2}^{\mathbf{e}}$ jour & $\mathbf{4}^{\mathbf{e}}$ jour & $\mathbf{6}^{\mathbf{e}}$ jour & $\mathbf{8}^{\mathbf{e}}$ jour \\
\hline Maradi & 9,19 & 39,75 & 65,51 & 89,08 \\
Gaya & 60,24 & 87,05 & 95,03 & 97,99 \\
Ayérou & 4,22 & 35,21 & 69,85 & 80,88 \\
Tchintabarden & 40,77 & 79,48 & 92,67 & 99,98 \\
\hline
\end{tabular}


Tableau 7: Variation du sex-ratio (R) de C. maculatus en fonction de l'origine géographique des souches.

\begin{tabular}{lccc}
\hline $\begin{array}{l}\text { Origine des } \\
\text { souches }\end{array}$ & $\begin{array}{c}\text { Nbr moyen de mâles émergés } \\
( \pm \text { Ecart type })\end{array}$ & $\begin{array}{c}\text { Nbr moyen de femelles émergées } \\
( \pm \text { Ecart type })\end{array}$ & $\begin{array}{c}\text { Sex-ration } \\
(\mathbf{R})\end{array}$ \\
\hline Maradi & $30,05 \pm 9,71 \mathrm{~b}$ & $23,5 \pm 9,33 \mathrm{~b}$ & 1,27 \\
Gaya & $15,7 \pm 5,39 \mathrm{a}$ & $17,9 \pm 4,81 \mathrm{a}$ & 0,87 \\
Ayerou & $13,65 \pm 4,77 \mathrm{a}$ & $14,35 \pm 6,33 \mathrm{a}$ & 0,95 \\
Tchinta & $16,5 \pm 7,75 \mathrm{a}$ & $13,4 \pm 6,26 \mathrm{a}$ & 1,23
\end{tabular}

Dans une même colonne, les moyennes suivies par la même lettre ne sont pas significativement différentes au seuil de $5 \%$ (Test de Newman-Keuls).

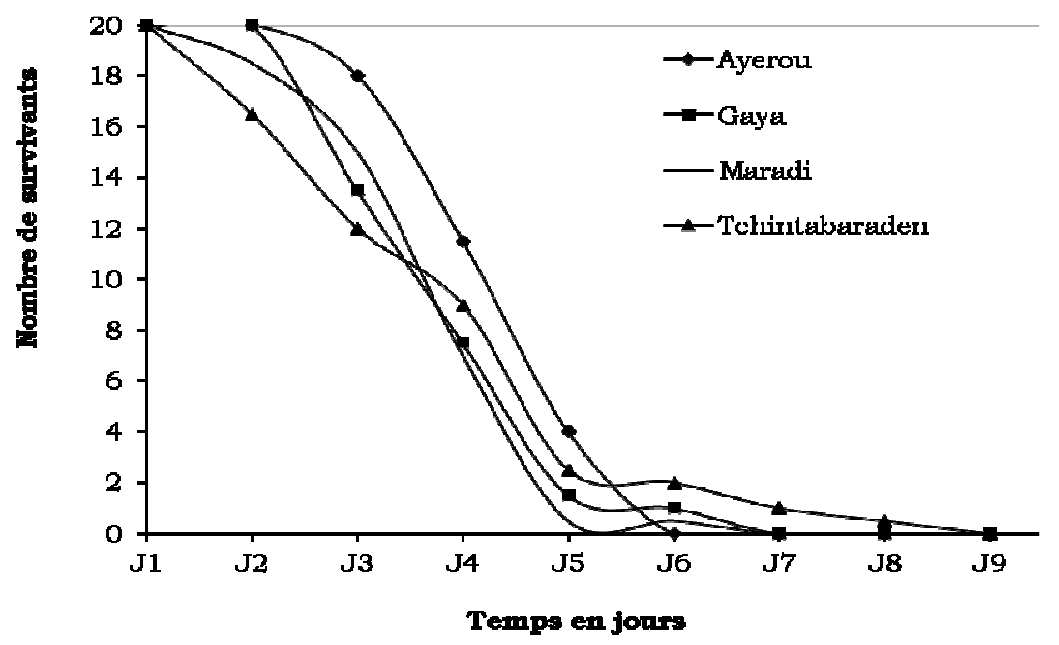

Figure 2: Evolution de la mortalité des adultes de C. maculatus en fonction du temps et de l'origine des souches.

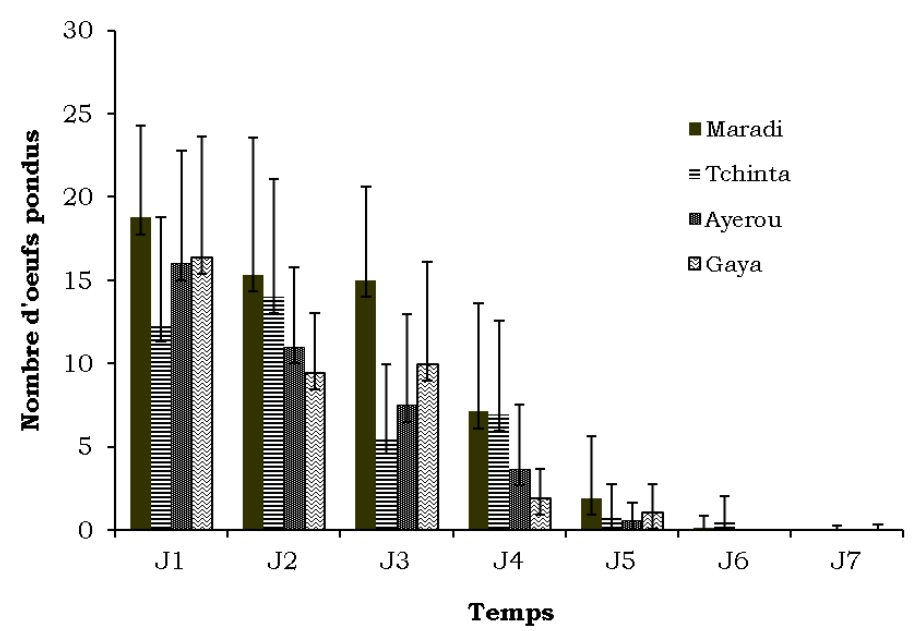

Figure 3 : Evolution du nombre d'œufs pondus en fonction du temps et de l'origine de la souche. 


\section{DISCUSSION}

Les résultats obtenus au cours de cette étude montrent que les paramètres biologiques de $C$. maculatus varient en fonction de l'origine agro-écologique de la souche. Dans nos conditions expérimentales, la durée de vie des adultes de cette espèce est courte et varie entre 4,67 et 5,65 jours. Ces résultats sont proches avec ceux observés par Sanon (1997) qui a observé une durée de vie moyenne de 6,4 jours dans les conditions naturelles au Burkina Faso et par fatimeh (2009) qui a observée une durée de vie de 5,87 jours dans les conditions $25 \pm 1{ }^{\circ} \mathrm{C}$ de température, $20 \pm 5 \%$ d'humidité relative et 16:8 (L: D) h de photopériode. Selon Howe et Curie (1964), les espèces du genre Callosobruchus ne vivent en général que quelques jours.

Par ailleurs, les résultats montrent que la longévité de $C$. maculatus est fonction de l'origine géographique de la souche. Les souches de Tarna et d'Ayérou ont enregistré les durées de vie les plus élevées. Ce résultat est surtout liée à la variation de la durée de vie des mâles puisque la durée de vie des femelles est la même pour toutes les souches. Mais la durée de vie de cette espèce de bruche augmente lorsqu'elle est nourri par les fleurs de certains arbres tels que $A$. seyal, $B$. aegyptiaca, N. macrophylla (Alfari, 2012). Dans ces conditions cet Auteur a trouvé une durée de vie qui tourne autour de 8 jours quelque soit les fleurs utilisées.

L'étude de l'activité de ponte de $C$. maculatus montre que le nombre d'œufs pondus par cette espèce varie en fonction de la souche utilisée. C'est ainsi que le nombre moyen d'œufs pondus est significativement plus important pour la souche de Tarna que pour les souches des autres localités. Cependant, quelle que soit la localité considérée, le taux de fertilité est important et est supérieur à $90 \%$ comme l'ont déjà indiqué Kellouche et al. (2004).
Cette forte activité de ponte expliquerait la durée de vie relativement brève des femelles observée au niveau des différentes souches. En effet, l'activité de ponte entraine une réduction significative de la durée de vie de la femelle en raison de la dépense des réserves accumulées sous forme de corps gras, pour la maturation des ovocytes lors de l'oviposition. Selon Tatar et al. (1993), l'accouplement affecte de manière significative la durée de vie d'une femelle. D'où le concept de «coût de la reproduction » utilisé par Williams (1966) pour relier l'effort de la reproduction aux autres fonctions de l'insecte.

Les résultats sur l'évolution de la fécondité moyenne journalière montrent que pour toutes les souches, l'essentiel des œufs est pondu pendant les quatre premiers jours de l'infestation comme cela a été observé chez de nombreux bruchidae. Ces résultats corroborent ceux observés par Nyamador (2009) chez C. subinnotatus qui a montré que la femelle de cette espèce dépose l'essentiel de ses œufs $(80,83 \%)$ en 6 jours avec un pic au 3 ème jour au cours d'une durée moyenne de vie de $11,36 \pm 1,85$ jours.

La durée de développement embryonnaire varie peu entre les quatre souches (moins de 5 jours). Cependant, la durée de développement totale montre des variations importantes en fonction des souches. Elle est relativement plus longue pour les souches de Gaya et d'Ayerou que pour celle de Tarna et de Tchintabaraden (33 contre 30 jours).

Le taux de survie larvaire et le taux d'émergence sont élevés et varient également selon la souche. Ils sont plus importants pour les souches de Tarna et de Gaya que pour celles d'Ayérou et de Tchintabaraden. Ces taux élevés sont favorisés par les conditions climatiques qui influencent le développement des larves et substrat de ponte qui limite le dépôt de plusieurs œufs sur la graine par la 
femelle qui évite ainsi un accroissement de la densité larvaire intragranaire. Selon les travaux de Zannou (2000), Booker (1967), Howe et Curie (1964), lorsque la densité larvaire intragranaire augmente, le taux de mortalité larvaire augmente également ; mais le taux d'émergence diminue.

Le sex-ratio varie aussi en fonction des souches. Il est en faveur des femelles pour les souches de Gaya et de Ayérou et en faveur des mâles pour les souches de Maradi et de Tchintabaraden.

Ainsi l'origine géographique des souches semble influencer les paramètres biologiques de $C$. maculatus dans nos conditions contrairement aux résultats observés par Attachi et al., (2003). En comparant les paramètres biologiques de cet insecte à partir de différentes combinaisons de facteurs liées à trois conditions thermohygrométriques $\left(32{ }^{\circ} \mathrm{C}\right.$ et $60 \%$ H.R., $27{ }^{\circ} \mathrm{C}$ et $70 \%$ H.R. puis $23{ }^{\circ} \mathrm{C}$ et $80 \%$ H.R), quatre substrats alimentaires (grains et les gousses de niébé (Vigna unguiculata (L.) Walp.) et de Cassia occidentalis) et deux souches de $C$. maculatus originaires du sud et du nord Bénin, ces auteurs ont montré que seul le substrat constitué de grains de niébé a entraîné une augmentation significative du taux net de reproduction (Ro ) et du taux d'accroissement intrinsèque ( $\mathrm{rm}$ ) de la population.

Les variations des paramètres biologiques observées dans nos conditions pourraient être liées soit directement à la souche qui semble mieux s'adapter aux conditions du milieu d'étude soit aux conditions de prélèvement des échantillons. En effet, les échantillons ayant été prélevés aux champs et chez des producteurs différents, il se pourrait que les gousses n'aient pas été récoltées à la même période.

\section{Conclusion}

Les résultats obtenus au cours de cette étude montrent que les souches nigériennes de
Callosobruchus maculatus présentent un potentiel reproducteur important. Par conséquent, la mise au point des méthodes de lutte susceptibles de réduire cette activité reproductrice est indispensable. Ces résultats représentent un bon indicateur pour les chercheurs car ils supposent qu'une période de ponte de quatre jours est largement suffisante pour évaluer l'activité reproductrice de C. maculatus.

\section{REFERENCES}

Alfari BY. 2012. Influence du régime alimentaire à base de fleurs de quatre espèces végétales sur les paramètres biologiques de Callosobruchus maculatus Fab. et Bruchidius atrolineatus Pic. (Coleaptera: Bruchinea), ravageurs du niébé (Vigna unguiculata Walp.). Laboratoire de biologie à la Faculté des Sciences et Technique de Niamey, p 30-40.

Alzouma I. 1995. Connaissance et contrôle des Coléoptères Bruchidæ ravageurs des légumineuses alimentaires au Sahel. Sahel IPM, 1(4): 10-11.

Alzouma I, Huignard J, Leroi B. 1985. Etude du comportement de ponte de Bruchidius atrolineatus (coleoptera, Bruchidae) au cours de la maturation des gousses de sa plante-hôte (Vigna unguiculata (L.) WALP.) et de ses conséquences sur le développement des œufs. Ann. Soc. Ent. Fr., 21: 207-217.

Atachi P, Kossou D, Tchéhouali N. 2003. Etude experimentale des parametres biologiques de callosobruchus maculatus Fab. (Coleoptera: bruchidae) sur Vigna unguiculata (1.) Walp. et sur Cassia occidentalis (linn.). Annale des Sciences Agronomiques du Bénin, 2(5): 342-351.

Booker RH. 1967. Observation on three Bruchids associated with cowpea in Northern Nigeria. J. Stored Prod. Res., 3: $1-15$.

Doumma A. 1998. Contribution à la recherche 
de méthodes de lutte contre Bruchidius atrolineatus Pic. Et Callosobruchus maculatus Fab. (Coleoptera: Bruchidæ), Ravageur de niébé ( $V$. unguiculata L. Walp) en zone sahélienne. Thèse de doctorat, Univ. Abdou Moumouni de Niamey, 135p.

Fatemeh K, Ali Asghar T, Yaghoub F, Samira F. 2009. A Comparative Study on the Effect of Four Leguminous Species on Biological and Population Growth Parameters of Callosobruchus maculatus (F.) (Coleoptera: Bruchidae). Adv. Environ. Biol., 3(3): 226-232.

Gatehouse AMR, Gathehouse JA, Dobie P, Kilminster, AM, Boulter, D. 1979. Biochemical basis of insect resistance in Vigna unguiculata. J. Sci. Food Agric., 30: 948-958.

Gauthier N. 1996. Etude d'un ectoparasitoïde, Dinarmus basalis Rond (Hymenopteraptéromalidae) en situation de compétition intra- et interspécifique: Activité reproductrice et réponses comportementales. Thèse de Doctorat, Université de Tours, 183 pages.

Glitho IA. 1990. Les Bruchidae ravageurs de Vigna unguiculata Walp. en zone guinéenne. Analyse de la diapause reproductrice chez les mâles de Bruchidius atrolineatus Pic. Thèse de Doctorat, Univ. F. Rabelais, Tours, 100 p.

Howe RC, Curie JF. 1964. Some laboratory observations of the rates of the development, mortality and oviposition of several species of bruchidae breeding in stored pulses. Hull. Ent. Res., 55: 437-477.

Kellouche A, Soltani N, Huignard J. 2004. Activité de reproduction et capacité de développement de la descendance de Callosobruchus maculatus (Fabricius) (Coleoptera: Bruchidae) dans des graines de différents cultivars de Vigna unguiculata (Walp.) et de Cicer arietinum
(L.). International Journal of Tropic Insects Science, 24(4): 304-310.

Lale NES, Vidal S. 2003. Effect of constant temperature and humidity on oviposition and development of Callosobruchus maculatus (F.) and Callosobruchus subinnotatus (Pic) on bambara groundnut, Vigna subterranea (L.) Verdcourt. J. Stored Prod. Res., 39: 459-470.

Monge JP, Germain JF, Huignard J. 1988. Importance des variations sur l'induction de la diapause reproductrice chez $B$. atrolineatus Pic (Coleoptera-Bruchidae). Acta oecol., Oecol. Apl., 9(3): 297-307.

Nyamador SW. 2009. Influence des traitements à base d'huiles essentielles sur les capacités de reproduction de Callosobruchus subinnotatus Pic. et de Callosobruchus maculatus F. (Coleoptera : Bruchidæ) : Mécanisme d'action de l'huile essentielle de Cymbopogon giganteus Chiov. Thèse de Doctorat, 197 pages.

Ouedraogo AP, Sou S, Sanon A, Monge J-P, Huignard J, Tran B, Credland PF. 1996. Influence of temperature and relative humidity on population of Callosobruchus maculatus (Coleoptera: Bruchidae) and its parasitoid Dinarmus basalis (Pteromalidae) in two climatic zones of Burkina Faso. Bull. Entomol. Res., 86: 695-702.

Ouedraogo PA. 1991. Le déterminisme du polymorphisme imaginal chez Callosobruchus maculatus (Fab.) (Coleoptera : Bruchidæ). Importances des facteurs climatiques sur l'évolution des populations de ce Bruchidæ dans un système expérimental de stockage de graines de Vigna unguiculata (Walp.) Thèse de Doctorat. Université de Côte d'Ivoire, 117p.

RECA. 2012. Réseau National des Chambres d'Agriculture du Niger, Note d'information / Actualités Niger n²5, http://www.recaniger.org/spip.php?article398 consulté le 24/06/2013. 
Sanon A. 1997. Contribution à l'étude du contrôle biologique des populations des bruchidae ravageurs de graines du niébé, $V$. unguiculata, au cours de leur stockage au Burkina Faso. Thèse de Doctorat, Univ. Ouagadougou, 162p.

Sanon A, Sou S, Dabire' LCB, Ouedraogo AP, Huignard J. 2005. Combining Boscia senegalensis Lam. (Capparaceae) leaves and augmentation and the larval parasitoid Dinarmus basalis Rondani (Hymenoptera: Pteromalidae) for bruchids control in stored cowpea. J. Entomol., 2(1): 40-45.

Sanon A, Ouedraogo PA, Tricault Y, Credland PF, Huignard J. 1998. Biological control of bruchids in cowpea stores by release of Dinarmus basalis (Hymenoptera: Pteromalidae) adults. Environmental Entomology, 27: 717-725.

Schoof HF. 1941. The effect of various relative humidities on the life processes of the southern Cowper weevil, Callosobruchus maculatus (F.) at $30{ }^{\circ} \mathrm{C} \pm$ 0,8. Ecology, 297-305.

Seck D, Sidibé B, Haubruge E, lienari V, Garpar C. 1992. La Résistance variétale du niébé (Vigna unguiculata (L) Walp.) à
Callosobruchus maculatus F. (ColeopteraBruchidæ): Evaluation et perspectives d'utilisation au Sénégal Med. Fac. Landbouww. Rijksuniv. Gent, 57(3a): 743750.

Tatar M, Carey JR, Vaupel JW. 1993. Long terme cost of reproduction with and without accelerated senescence in Callosobruchus maculatus: Analyse of agespecific mortality. Evolution, 47: 13051312.

Wellington WG. 1977. Returning the insect to insect ecology: some consequences for pest management. Env. Entomol., 6: 1-8.

Williams GC. 1966. Natural selection, the costs of reproduction and a refinement of lack's principle. Amer. Natur., 100: 687690.

Zannou ET. 2000. Analyse de quelques paramètres biologiques pour une meilleure connaissance des capacités reproductrices d'une souche béninoise de Callosobruchus maculatus F. (Coleoptera : Bruchidae) : caractérisation et importance de la diapause reproductrice chez les femelles du morphe voilier. Thèse de Doctorat, Univ. Bénin Lomé, Togo, 122 p. 